\title{
DIFFERENTIABILITY OF THE SCALAR COEFFICIENTS IN TWO REPRESENTATION FORMULAE FOR ISOTROPIC TENSOR FUNCTIONS IN TWO DIMENSIONS
}

\author{
BY \\ CHI-SING MAN AND JEFFREY B. SCHANDING \\ Department of Mathematics, University of Kentucky, Lexington, $K Y$
}

1. Introduction. While very general representation theorems have been proved for isotropic tensor functions [1-3], almost all of these theorems are algebraic in character. Take the most familiar representation formula in (three-dimensional) continuum mechanics for example, namely

$$
\mathbf{T}(\mathbf{B})=\gamma_{1} \mathbf{I}+\gamma_{2} \mathbf{B}+\gamma_{3} \mathbf{B}^{2}
$$

here $\mathbf{T}:$ Sym $\rightarrow$ Sym is a given isotropic tensor function; Sym denotes the set of symmetric second-order tensors, and $\mathbf{I}$ is the identity tensor. The algebraic theorem asserts that for each isotropic function $\mathbf{T}(\cdot)$, there are coefficients $\gamma_{1}, \gamma_{2}$, and $\gamma_{3}$, which are scalar functions of the principal invariants of $\mathbf{B}$, such that Eq. (1.1) is valid for each $\mathbf{B}$ in Sym. Moreover, it is well known that the coefficients $\gamma_{i}$ are uniquely determined by Eq. (1.1) only if the eigenvalues of $\mathbf{B}$ are distinct.

A natural question on the continuity and differentiability properties of $\gamma_{i}$ arises: suppose $\mathbf{T}(\cdot)$ is of class $C^{n} \quad(n \geq 0)$; can we choose the coefficients $\gamma_{i}$ so that they are of class $C^{k} \quad(0 \leq k \leq n)$ ? In 1959, Serrin [4] gave a counterexample in which $\mathbf{T}(\cdot)$ is differentiable but there is no way to choose the $\gamma_{i}$ such as to remain continuous. He went on to prove in the same article that the $\gamma_{i}$ can be chosen to be continuous if $\mathbf{T}(\cdot)$ is of class $C^{3}$. Recently Man [5] improved Serrin's sufficient condition of smoothness from $C^{3}$ to $C^{2}$, but that is all that we currently know about the analytical aspects of the representation formula (1.1). ${ }^{1}$ Except for the analogue of (1.1) in two-dimensional space (cf. Man [5]) and a major memoir of Ball [6] on scalar-valued functions, as far as we are aware, nothing else has been published on the analytical aspects of other representation formulae for isotropic tensor functions.

As an explanation for such a deplorable situation, there is the argument that the continuity and differentiability of representation formulae are ignored because they are irrelevant in many applications. We do not accept this argument. Even if we set

Received September 24, 1993.

1991 Mathematics Subject Classification. Primary 73B05, 15A72, 15 A90.

${ }^{1}$ Note added in proof. Man [12] has recently proved two theorems on the smoothness of the scalar coefficients in the representation formula (1.1). 
aside the intrinsic merit in delineating the continuity and differentiability properties, it is a fact that those properties are crucial in some applications (cf. the last paragraph of this section).

In this paper we study the smoothness of scalar coefficients in two representation formulae for isotropic tensor functions in two-dimensional space. The first formula concerns isotropic functions $\mathbf{H}: \operatorname{Sym} \times \operatorname{Sym} \rightarrow \operatorname{Sym},(\mathbf{A}, \mathbf{E}) \mapsto \mathbf{H}(\mathbf{A}, \mathbf{E})$, which are linear in $\mathbf{E}$ :

$$
\mathbf{H}(\mathbf{A}, \mathbf{E})=\left(\alpha_{11} \operatorname{tr} \mathbf{E}+\alpha_{12} \operatorname{tr}(\mathbf{A E})\right) \mathbf{I}+\left(\alpha_{21} \operatorname{tr} \mathbf{E}+\alpha_{22} \operatorname{tr}(\mathbf{A E})\right) \mathbf{A}+\alpha_{3} \mathbf{E},
$$

where $\mathbf{I}$ is the two-dimensional identity tensor and the coefficients $\alpha_{i j}$ and $\alpha_{3}$ are symmetric functions of the repeated eigenvalues of $\mathbf{A}$. Appealing to the work of Rivlin and Ericksen [7], Noll [8] first wrote down the analogue of the representation formula (1.2) in three-dimensional space. Here we show in Theorem 3.1 below that if $\mathbf{H}$ is of class $C^{n+2}(n \geq 0)$, then there is a unique choice of coefficients in (1.2) for which the $\alpha_{i j}$ are of class $C^{n}$ and $\alpha_{3}$ is of class $C^{n+2}$.

The mathematical apparatus by which we derive the aforementioned differentiability properties of scalar coefficients in (1.2) applies also to the representation formula

$$
\mathbf{G}(\mathbf{A})=\beta_{1} \mathbf{I}+\beta_{2} \mathbf{A}
$$

for an isotropic function $\mathbf{G}: \mathrm{Sym} \rightarrow \mathrm{Sym}$ in two-dimensional space, where $\beta_{1}$ and $\beta_{2}$ are symmetric functions of the repeated eigenvalues of $\mathbf{A}$. We show that if $\mathbf{G}$ is of class $C^{n+1} \quad(n \geq 0)$, then we may choose $\beta_{1}$ and $\beta_{2}$ in (1.3) so that they are of class $C^{n}$ (see Theorem 3.3).

The class of isotropic tensor functions given by (1.2) above has various mathematical and physical applications. For instance, if the isotropic function $\mathbf{G}: \mathrm{Sym} \rightarrow$ Sym, $\mathbf{A} \mapsto \mathbf{G}(\mathbf{A})$, is of class $C^{n+3} \quad(n \geq 0)$, then its derivative $D \mathbf{G}(\mathbf{A})$ is a linear transformation on Sym and is of class $C^{n+2}$ in A. Equation (1.2) will then deliver a basis-free formula for $D \mathbf{G}(\mathbf{A})[\cdot]$. To mention one physical application of (1.2), consider metal sheets that have null prestress and are prepared from some isotropic natural state by plastic prestraining. If we restrict our attention to small in-plane elastic motions superimposed on such sheets, then (1.2) will be appropriate for modelling the constitutive equation of the sheets [9]. In this context, A will denote the plastic Karni-Reiner prestrain, $\mathbf{E}$ the incremental (infinitesimal) elastic strain, and $\mathbf{H}$ the surface elasticity tensor. It turns out [9] that the coefficient $\alpha_{22}$ in (1.2) is related to the "acoustoelastic earing coefficient" of the plastically prestrained sheets and its smoothness is crucial in the analysis of some physical problems.

2. Mathematical preliminaries. First we prove two theorems on symmetric functions that we shall need later. Their proof is based on Lemmas 2.1 and 2.2 below. After we constructed our proof of these lemmas, we noticed Whitney's 1943 paper on "Differentiability of the remainder term in Taylor's formula" [10]. Our lemmas are in essence nothing but a slightly reworded version of some of Whitney's results. As far as these two lemmas go, however, we believe that our proof is simpler than the original proof of Whitney's, which covers more ground than what concerns us here. 
For completeness and for ease of reading, we decide not to appeal to Whitney's work but present our independent proof of Lemmas 2.1 and 2.2.

Lemma 2.1. Let $f: \mathbb{R}^{2} \rightarrow \mathbb{R}^{1}$, where $f \in C^{m}$ and $m \geq 1$. Define

$$
f_{m}(x, y)= \begin{cases}\left(f(x, y)-\sum_{r=0}^{m-1} \frac{\partial^{r} f}{\partial y^{r}}(x, 0) y^{r} / r !\right) / y^{m}, & \text { when } y \neq 0, \\ \frac{\partial^{m} f}{\partial y^{m}}(x, 0) / m !, & \text { when } y=0 .\end{cases}
$$

Then $f_{m}$ is continuous, and

$$
\lim _{\substack{(x, y) \rightarrow\left(x_{0}, 0\right) \\ y \neq 0}} \frac{\partial^{k} f_{m}}{\partial y^{k}}(x, y) y^{k}=0 \text { for } 1 \leq k \leq m .
$$

Proof. For $y \neq 0$, since

$$
\begin{aligned}
\left(f(x, y)-\sum_{r=0}^{m-1} \frac{\partial^{r} f}{\partial y^{r}}(x, 0) y^{r} / r !\right) / y^{m} & =\left(\frac{\partial^{m} f}{\partial y^{m}}\left(x, y^{\prime}\right) y^{m} / m !\right) / y^{m} \\
& =\frac{\partial^{m} f}{\partial y^{m}}\left(x, y^{\prime}\right) / m !
\end{aligned}
$$

for some $y^{\prime}$ with $\left|y^{\prime}\right|<|y|$, continuity of $\partial^{m} f / \partial y^{m}$ yields

$$
\begin{aligned}
\lim _{\substack{(x, y) \rightarrow\left(x_{0}, 0\right) \\
y \neq 0}} f_{m}(x, y) & =\lim _{\substack{(x, y) \rightarrow\left(x_{0}, 0\right) \\
y \neq 0}} \frac{\partial^{m} f}{\partial y^{m}}\left(x, y^{\prime}\right) / m ! \\
& =\frac{\partial^{m} f}{\partial y^{m}}\left(x_{0}, 0\right) / m ! .
\end{aligned}
$$

For $y=0$, it is clear that

$$
\lim _{(x, 0) \rightarrow\left(x_{0}, 0\right)} \frac{\partial^{m} f}{\partial y^{m}}(x, 0)=\frac{\partial^{m} f}{\partial y^{m}}\left(x_{0}, 0\right) .
$$

Hence $f_{m}$ is continuous. We now proceed to prove the lemma by induction. For $m=1$ and $y \neq 0$, we have $f(x, y)=f_{1}(x, y) y+f(x, 0)$. Differentiating boih sides of the preceding equation with respect to $y$, we obtain

$$
\frac{\partial f}{\partial y}(x, y)=\frac{\partial f_{1}}{\partial y}(x, y) y+f_{1}(x, y) .
$$

By the continuity of $f_{1}$ and of $\partial f / \partial y$, we conclude that

$$
\lim _{\substack{(x, y) \rightarrow\left(x_{0}, 0\right) \\ y \neq 0}} \frac{\partial f_{1}}{\partial y}(x, y) y=0 .
$$

For $m \geq 2$, we write $m=n+2$, where $n \geq 0$. For $y \neq 0$, since

$$
f(x, y)=f_{n+2}(x, y) y^{n+2}+\sum_{r=0}^{n+1} \frac{\partial^{r} f}{\partial y^{r}}(x, 0) y^{r} / r !,
$$


we have

$$
\frac{\partial f}{\partial y}(x, y)=(n+2) f_{n+2}(x, y) y^{n+1}+\frac{\partial f_{n+2}}{\partial y}(x, y) y^{n+2}+\sum_{r=0}^{n} \frac{\partial^{r}}{\partial y^{r}}\left(\frac{\partial f}{\partial y}\right)(x, 0) y^{r} / r !,
$$

and

$$
\left(\frac{\partial f}{\partial y}(x, y)-\sum_{r=0}^{n} \frac{\partial^{r}}{\partial y^{r}}\left(\frac{\partial f}{\partial y}\right)(x, 0) y^{r} / r !\right) / y^{n+1}=(n+2) f_{n+2}(x, y)+\frac{\partial f_{n+2}}{\partial y}(x, y) y .
$$

Hence

$$
\frac{\partial^{n+2} f}{\partial y^{n+2}}\left(x, y^{\prime}\right) /(n+1) !-(n+2) f_{n+2}(x, y)=\frac{\partial f_{n+2}}{\partial y}(x, y) y,
$$

for some $y^{\prime}$ with $\left|y^{\prime}\right|<|y|$. By taking the limit as $(x, y) \rightarrow\left(x_{0}, 0\right)$ on both sides, we obtain

$$
\lim _{\substack{(x, y) \rightarrow\left(x_{0}, 0\right) \\ y \neq 0}} \frac{\partial f_{n+2}}{\partial y}(x, y) y=0 .
$$

Thus the lemma is valid for $k=1$. Now suppose the lemma holds up to $k=p-1$. From

$$
f(x, y)=f_{n+2}(x, y) y^{n+2}+\sum_{r=0}^{n+1} \frac{\partial^{r} f}{\partial y^{r}}(x, 0) y^{r} / r !
$$

we obtain for $y \neq 0$ and $2 \leq p \leq n+1$

$$
\begin{aligned}
\frac{\partial^{p} f}{\partial y^{p}}(x, y)= & \sum_{r=0}^{p} \frac{(n+2) !}{(n+2-r) !} C_{r}^{p} \frac{\partial^{p-r} f_{n+2}}{\partial y^{p-r}}(x, y) y^{n+2-r} \\
& +\sum_{r=0}^{n+1-p} \frac{\partial^{r}}{\partial y^{r}}\left(\frac{\partial^{p} f}{\partial y^{p}}\right)(x, 0) y^{r} / r !
\end{aligned}
$$

where $C_{r}^{p}=p ! /(p-r) ! r !$. It follows that

$$
\begin{gathered}
\left(\frac{\partial^{p} f}{\partial y^{p}}(x, y)-\sum_{r=0}^{n+1-p} \frac{\partial^{r}}{\partial y^{r}}\left(\frac{\partial^{p} f}{\partial y^{p}}\right)(x, 0) y^{r} / r !\right) / y^{n+2-p} \\
=\frac{1}{y^{n+2-p}}\left(\sum_{r=0}^{p} \frac{(n+2) !}{(n+2-r) !} C_{r}^{p} \frac{\partial^{p-r} f_{n+2}}{\partial y^{p-r}}(x, y) y^{n+2-r}\right)
\end{gathered}
$$

Hence, for some $y^{\prime}$ with $\left|y^{\prime}\right|<|y|$,

$$
\frac{\partial^{n+2} f}{\partial y^{n+2}}\left(x, y^{\prime}\right) /(n+2-p) !=\sum_{r=0}^{p} \frac{(n+2) !}{(n+2-r) !} C_{r}^{p} \frac{\partial^{p-r} f_{n+2}}{\partial y^{p-r}}(x, y) y^{p-r} \text {, }
$$


which implies

$$
\begin{aligned}
& \frac{\partial^{n+2} f}{\partial y^{n+2}}\left(x, y^{\prime}\right) /(n+2-p) ! \\
& =\frac{\partial^{p} f_{n+2}}{\partial y^{p}}(x, y) y^{p}+\frac{(n+2) !}{(n+2-p) !} f_{n+2}(x, y) \\
& \quad+\sum_{r=1}^{p-1} \frac{(n+2) !}{(n+2-r) !} C_{r}^{p} \frac{\partial^{p-r} f_{n+2}}{\partial y^{p-r}}(x, y) y^{p-r}
\end{aligned}
$$

By taking the limit on each side as $(x, y) \rightarrow\left(x_{0}, 0\right)$, we have

$$
\lim _{\substack{(x, y) \rightarrow\left(x_{0}, 0\right) \\ y \neq 0}} \frac{\partial^{p} f_{n+2}}{\partial y^{p}}(x, y) y^{p}=0
$$

for $2 \leq p \leq n+1$. For $p=n+2$, instead of Eq. (2.14) we have for $y \neq 0$,

$$
\begin{aligned}
\frac{\partial^{n+2} f}{\partial y^{n+2}}(x, y)= & \sum_{r=0}^{n+2} \frac{(n+2) !}{(n+2-r) !} C_{r}^{n+2} \frac{\partial^{n+2-r} f_{n+2}}{\partial y^{n+2-r}}(x, y) y^{n+2-r} \\
= & \frac{\partial^{n+2} f_{n+2}}{\partial y^{n+2}(x, y) y^{n+2}+(n+2) ! f_{n+2}(x, y)} \\
& +\sum_{r=1}^{n+1} \frac{(n+2) !}{(n+2-r) !} C_{r}^{n+2} \frac{\partial^{n+2-r} f_{n+2}}{\partial y^{n+2-r}}(x, y) y^{n+2-r} .
\end{aligned}
$$

Taking the limit on both sides of Eq. (2.19) as $(x, y) \rightarrow\left(x_{0}, 0\right)$, we deduce that

$$
\lim _{\substack{(x, y) \rightarrow\left(x_{0}, 0\right) \\ y \neq 0}} \frac{\partial^{n+2} f}{\partial y^{n+2}}(x, y) y^{n+2}=0 .
$$

Lemma 2.2. Let $f: \mathbb{R}^{2} \rightarrow \mathbb{R}^{1}$ be a $C^{n+k}$ function $(n \geq 0, k \geq 1)$ that satisfies $f(x, 0)=0$ and $(\partial f / \partial y)(x, 0)=\cdots=\left(\partial^{k-1} f / \partial y^{k-1}\right)(x, 0)=0$ for all $x$. Define

$$
g(x, y)= \begin{cases}f(x, y) / y^{k}, & \text { when } y \neq 0 \\ \frac{\partial^{k} f}{\partial y^{k}}(x, 0) / k ! & \text { when } y=0\end{cases}
$$

Then $g \in C^{n}\left(\mathbb{R}^{2}\right)$, and

$$
\frac{\partial^{p} g}{\partial y^{p}}(x, 0)=\frac{p !}{(p+k) !} \frac{\partial^{p+k} f}{\partial y^{p+k}}(x, 0) \text { for } 0 \leq p \leq n .
$$

Proof. Since $g=f_{k}$, we conclude from Lemma 2.1 that $g \in C^{0}\left(\mathbb{R}^{2}\right)$. For $y \neq$ 0 and $0<p \leq n, \partial^{p} g / \partial x^{p}=\left(\partial^{p} f / \partial x^{p}\right) / y^{k}$, where $\partial^{p} f / \partial x^{p} \in C^{n+k-p}$ and $\left(\partial^{p} f / \partial x^{p}\right)(x, 0)=0,\left(\partial^{l}\left(\partial^{p} f / \partial x^{p}\right) / \partial y^{l}\right)(x, 0)=\left(\partial^{p}\left(\partial^{l} f / \partial y^{l}\right) / \partial x^{p}\right)(x, 0)=0$ for $1 \leq l \leq k-1$. Hence it follows from Lemma 2.1 again that $\partial^{p} g / \partial x^{p} \in C^{0}\left(\mathbb{R}^{2}\right)$ if we define $\left(\partial^{p} g / \partial x^{p}\right)(x, 0)=\left[\left(\partial^{k}\left(\partial^{p} f / \partial x^{p}\right) / \partial y^{k}\right)(x, 0)\right] / k !$. 
Now we consider $\partial^{p} g / \partial y^{p}$. For $y \neq 0$, by Eq. (2.1) we recast Eq. (2.21) as

$$
g(x, y)=\frac{f(x, y)}{y^{k}}=f_{n+k}(x, y) y^{n}+\sum_{r=0}^{n-1} \frac{\partial^{r+k} f}{\partial y^{r+k}}(x, 0) \frac{y^{r}}{(r+k) !} .
$$

Then, for $1 \leq p \leq n-1$,

$$
\begin{aligned}
\frac{\partial^{p} g}{\partial y^{p}}(x, y)= & \sum_{r=0}^{p} C_{r}^{p} \frac{n !}{(n-r) !} \frac{\partial^{p-r} f_{n+k}}{\partial y^{p-r}}(x, y) y^{n-r} \\
& +\sum_{r=0}^{n-1-p} \frac{\partial^{r+p+k} f}{\partial y^{r+p+k}}(x, 0) \frac{(p+r) ! y^{r}}{r !(p+k+r) !} .
\end{aligned}
$$

By Lemma 2.1, we conclude that

$$
\lim _{\substack{(x, y) \rightarrow\left(x_{0}, 0\right) \\ y \neq 0}} \frac{\partial^{p} g}{\partial y^{p}}=\frac{p !}{(p+k) !} \frac{\partial^{p+k} f}{\partial y^{p+k}}\left(x_{0}, 0\right),
$$

for $1 \leq p \leq n-1$. For $p=n$, we obtain from Eq. (2.23) that

$$
\frac{\partial^{n}}{\partial y^{n}}=\sum_{r=0}^{n} C_{r}^{n} \frac{n !}{(n-r) !} \frac{\partial^{n-r} f_{n+k}}{\partial y^{n-r}}(x, y) y^{n-r} .
$$

It follows from Lemma 2.1 that

$$
\begin{aligned}
\lim _{\substack{(x, y) \rightarrow\left(x_{0}, 0\right) \\
y \neq 0}} \frac{\partial^{n} g}{\partial y^{n}} & =n ! f_{n+k}\left(x_{0}, 0\right) \\
& =\frac{n !}{(n+k) !} \frac{\partial^{n+k} f}{\partial y^{n+k}}\left(x_{0}, 0\right) .
\end{aligned}
$$

It remains to consider the mixed partial derivatives of $g$. We note that for $y \neq 0$, $g \in C^{n+k}$; so we can do the partial differentiation in any order. In particular, a $p$ th-order mixed partial derivative, where $2 \leq p \leq n$, can be written as $\partial^{p-q}\left(\partial^{q} g / \partial x^{q}\right) / \partial y^{p-q}$ for some $1 \leq q<p$. But

$$
\frac{\partial^{p-q}}{\partial y^{p-q}}\left(\frac{\partial^{q} g}{\partial x^{q}}\right)=\frac{\partial^{p-q}}{\partial y^{p-q}}\left(\frac{1}{y^{k}} \frac{\partial^{q} f}{\partial x^{q}}\right)
$$

for $y \neq 0$. Moreover, since $\left(1 / y^{k}\right)\left(\left(\partial^{q} f / \partial x^{q}\right)(x, y)\right)$ is $C^{n+k-q}$ away from $y=0$ and

$$
\frac{\partial^{q} f}{\partial x^{q}}(x, 0)=\frac{\partial}{\partial y}\left(\frac{\partial^{q} f}{\partial x^{q}}\right)(x, 0)=\cdots=\frac{\partial^{k-1}}{\partial y^{k-1}}\left(\frac{\partial^{q} f}{\partial x^{q}}\right)(x, 0)=0
$$

for all $x$, its $(p-q)$ th partial derivative with respect to $y$ may be extended to all of $\mathbb{R}^{2}$ as above.

Finally, since $g \in C^{0}\left(\mathbb{R}^{2}\right), g \in C^{n}\left(\mathbb{R}^{2} \backslash\{(x, y): y=0\}\right)$ and since all the partial derivatives of $g$ of order 1 through $n$ can be continuously extended to all of $\mathbb{R}^{2}$, it follows from a lemma due to J. M. Ball ([6], Proposition 2.2) that $g \in C^{n}\left(\mathbb{R}^{2}\right)$. 
In what follows we shall use Lemma 2.2 only for the cases $k=1$ and $k=2$. We easily obtain the following theorems from Lemma 2.2 by a change of variables.

THEOREM 2.3. Let $f: \mathbb{R}^{2} \rightarrow \mathbb{R}^{1}$ be symmetric, $f \in C^{n+2}, n \geq 0$, and $f(\lambda, \lambda)=0$ for each $\lambda \in \mathbb{R}^{1}$. Then there exists a unique symmetric function $g: \mathbb{R}^{2} \rightarrow \mathbb{R}^{1}$, $g \in C^{n}$, such that $f\left(\lambda_{1}, \lambda_{2}\right)=g\left(\lambda_{1}, \lambda_{2}\right)\left(\lambda_{1}-\lambda_{2}\right)^{2}$ for all $\left(\lambda_{1}, \lambda_{2}\right) \in \mathbb{R}^{2}$.

Proof. Letting $x=\lambda_{1}+\lambda_{2}, y=\lambda_{1}-\lambda_{2}$, we define $F(x, y)=f((x+y) / 2$, $(x-y) / 2)$. Then $F \in C^{n+2}, F(x, 0)=f(x / 2, x / 2)=0$, and $F(x, y)=$ $f((x+y) / 2, \quad(x-y) / 2)=f((x-y) / 2, \quad(x+y) / 2)=F(x,-y) . \quad$ Thus, $(\partial F / \partial y)(x, 0)=0$. Now define

$$
G(x, y)= \begin{cases}F(x, y) / y^{2}, & \text { when } y \neq 0, \\ \frac{\partial^{2} F}{\partial y^{2}}(x, 0) / 2, & \text { when } y=0 .\end{cases}
$$

Then $G$ is of class $C^{n}$ by Lemma 2.2 , and $G(x, y)=G(x,-y)$. Define $g\left(\lambda_{1}, \lambda_{2}\right)=$ $g((x+y) / 2,(x-y) / 2)=G(x, y)$. Then $g \in C^{n}, g$ is symmetric, and $f\left(\lambda_{1}, \lambda_{2}\right)=$ $F(x, y)=G(x, y) y^{2}=g\left(\lambda_{1}, \lambda_{2}\right)\left(\lambda_{1}-\lambda_{2}\right)^{2}$.

THEOREM 2.4. Let $f: \mathbb{R}^{2} \rightarrow \mathbb{R}^{1}$ be symmetric (resp. alternating), $f \in C^{n+1}, n \geq 0$, and $f(\lambda, \lambda)=0$ for each $\lambda \in \mathbb{R}^{1}$. Then there exists a unique alternating (resp. symmetric) function $h: \mathbb{R}^{2} \rightarrow \mathbb{R}^{1}, h \in C^{n}$, such that $f\left(\lambda_{1}, \lambda_{2}\right)=h\left(\lambda_{1}, \lambda_{2}\right)\left(\lambda_{1}-\lambda_{2}\right)$ for all $\left(\lambda_{1}, \lambda_{2}\right) \in \mathbb{R}^{2}$.

Proof. We use the variables $x, y$ and define the function $F$ as in the proof of Theorem 2.3. Here $F \in C^{n+1}$. Let

$$
H(x, y)= \begin{cases}F(x, y) / y, & \text { when } y \neq 0, \\ \frac{\partial F}{\partial y}(x, 0), & \text { when } y=0 .\end{cases}
$$

Then, by Lemma 2.2, $H$ is of class $C^{n}$. Define $h\left(\lambda_{1}, \lambda_{2}\right)=h((x+y) / 2,(x-y) / 2)$ $=H(x, y)$. Then $h \in C^{n}, f\left(\lambda_{1}, \lambda_{2}\right)=F(x, y)=H(x, y) y=h\left(\lambda_{1}, \lambda_{2}\right)\left(\lambda_{1}-\lambda_{2}\right)$, and $h$ is alternating (resp. symmetric) if $f$ is symmetric (resp. alternating).

3. Two representation theorems. We proceed to prove our main theorem.

Theorem 3.1. Let Sym be the set of second-order symmetric tensors in two-dimensional space. Let $\mathbf{H}: \operatorname{Sym} \times \operatorname{Sym} \rightarrow \operatorname{Sym}, \mathbf{H}=\mathbf{H}(\mathbf{A}, \mathbf{E})$, and $\mathbf{H}$ be of class $C^{n+2}$. Let $\mathbf{H}$ be isotropic in $\mathbf{A}$ and $\mathbf{E}$; that is,

$$
\mathbf{H}\left(\mathbf{Q A Q}^{\mathrm{T}}, \mathbf{Q E Q}^{\mathrm{T}}\right)=\mathbf{Q H}(\mathbf{A}, \mathbf{E}) \mathbf{Q}^{\mathrm{T}}
$$

for each orthogonal $\mathbf{Q}$. Also, let $\mathbf{H}$ be linear in $\mathbf{E}$. Then there exist four $C^{n}$ functions $\alpha_{11}, \alpha_{12}, \alpha_{21}, \alpha_{22}$, and a $C^{n+2}$ function $\alpha_{3}: \mathbb{R}^{2} \rightarrow \mathbb{R}^{1}$, each of which is a symmetric function of the repeated eigenvalues of $\mathbf{A}$, such that

$$
\mathbf{H}(\mathbf{A}, \mathbf{E})=\left(\alpha_{11} \operatorname{tr} \mathbf{E}+\alpha_{12} \operatorname{tr}(\mathbf{A E})\right) \mathbf{I}+\left(\alpha_{21} \operatorname{tr} \mathbf{E}+\alpha_{22} \operatorname{tr}(\mathbf{A E})\right) \mathbf{A}+\alpha_{3} \mathbf{E} .
$$


Moreover, coefficient functions that satisfy Eq. (3.2) and enjoy the aforementioned continuity $(n=0)$ or differentiability $(n \geq 1)$ properties are unique.

Proof. For tensors $\mathbf{A}$ and $\mathbf{E}$ such that (i) $\mathbf{A}$ has distinct eigenvalues and (ii) $\mathbf{A}, \mathbf{E}$ do not share the same eigenvectors, the tensors $\mathbf{I}, \mathbf{A}$, and $\mathbf{E}$ are linearly independent. For the moment we consider only such $\mathbf{A}$ and $\mathbf{E}$. Under these circumstances we may express

$$
\mathbf{H}(\mathbf{A}, \mathbf{E})=\alpha_{1}(\mathbf{A}, \mathbf{E}) \mathbf{I}+\alpha_{2}(\mathbf{A}, \mathbf{E}) \mathbf{A}+\alpha_{3}(\mathbf{A}, \mathbf{E}) \mathbf{E}
$$

for some scalar-valued functions $\alpha_{i}(i=1,2,3)$. Since $\mathbf{H}$ is isotropic in $\mathbf{A}$ and $\mathbf{E}$, it will be convenient to choose a Cartesian coordinate system and restrict our attention to such $\mathbf{A}$ and $\mathbf{E}$ with matrix representation of the form

$$
\mathbf{A}=\left(\begin{array}{cc}
\lambda_{1} & 0 \\
0 & \lambda_{2}
\end{array}\right), \quad \mathbf{E}=\left(\begin{array}{ll}
e_{11} & e_{12} \\
e_{12} & e_{22}
\end{array}\right)
$$

where $\lambda_{1} \neq \lambda_{2}$ and $e_{12} \neq 0$. After we draw a conclusion, we can easily rewrite it in a coordinate-free way.

For all $\lambda \in \mathbb{R}^{1}, \alpha_{1}(\mathbf{A}, \lambda \mathbf{E}) \mathbf{I}+\alpha_{2}(\mathbf{A}, \lambda \mathbf{E}) \mathbf{A}+\alpha_{3}(\mathbf{A}, \lambda \mathbf{E})(\lambda \mathbf{E})=\mathbf{H}(\mathbf{A}, \lambda \mathbf{E})=\lambda \mathbf{H}(\mathbf{A}, \mathbf{E})$ $=\lambda \alpha_{1}(\mathbf{A}, \mathbf{E}) \mathbf{I}+\lambda \alpha_{2}(\mathbf{A}, \mathbf{E}) \mathbf{A}+\lambda \alpha_{3}(\mathbf{A}, \mathbf{E}) \mathbf{E}$. Since $\mathbf{I}, \mathbf{A}$, and $\mathbf{E}$ are linearly independent, $\alpha_{3}(\mathbf{A}, \lambda \mathbf{E}) \lambda=\lambda \alpha_{3}(\mathbf{A}, \mathbf{E})$. So $\alpha_{3}(\mathbf{A}, \lambda \mathbf{E})=\alpha_{3}(\mathbf{A}, \mathbf{E})$ for all $\lambda \neq 0$. Now consider $\mathbf{A}$ and $\mathbf{E}$ of the form given in (3.4). By a similar argument, if $\mathbf{E}^{\prime}$ is diagonal, then $\alpha_{3}(\mathbf{A}, \mathbf{E}) \cdot e_{12}+\alpha_{3}\left(\mathbf{A}, \mathbf{E}^{\prime}\right) \cdot 0=\alpha_{3}\left(\mathbf{A}, \mathbf{E}+\mathbf{E}^{\prime}\right) e_{12} ;$ so $\alpha_{3}$ is constant for a given value of $e_{12}$ and a fixed $\mathbf{A}$. Combining these two facts, we have $\alpha_{3}(\mathbf{A}, \cdot)$ constant over all $\mathbf{E}$ that do not share the same eigenvectors with $\mathbf{A}$.

We may extend the functions $\alpha_{3}(\mathbf{A}, \cdot)$ by continuity so that they are constant over Sym. By this choice we henceforth write $\alpha_{3}=\alpha_{3}(\mathbf{A})$, which is well defined for those $\mathbf{A}$ with distinct eigenvalues.

Let $\mathbf{G}(\mathbf{A}, \mathbf{E})=\mathbf{H}(\mathbf{A}, \mathbf{E})-\alpha_{3}(\mathbf{A}) \mathbf{E}$. From Eq. (3.3) we see that $\mathbf{G}$ and $\mathbf{A}$ have the same eigenvectors. Since $\mathbf{G}$ is well defined for all $\mathbf{E} \in \operatorname{Sym}$ and for all $\mathbf{A}$ with distinct eigenvalues, $\alpha_{1}$ and $\alpha_{2}$ are uniquely determined by Eq. (3.3) for all such $\mathbf{A}$ and $\mathbf{E}$. Since $\mathbf{H}$ is isotropic, we have

$$
\begin{aligned}
& \alpha_{1}\left(\mathbf{Q A Q} \mathbf{Q}^{\mathrm{T}}, \mathbf{Q E Q}^{\mathrm{T}}\right) \mathbf{I}+\alpha_{2}\left(\mathbf{Q A Q} \mathbf{Q}^{\mathrm{T}}, \mathbf{Q E E} \mathbf{Q}^{\mathrm{T}}\right) \mathbf{A}+\alpha_{3}\left(\mathbf{Q A} \mathbf{Q}^{\mathrm{T}}\right) \mathbf{E} \\
& =\alpha_{1}(\mathbf{A}, \mathbf{E}) \mathbf{I}+\alpha_{2}(\mathbf{A}, \mathbf{E}) \mathbf{A}+\alpha_{3}(\mathbf{A}) \mathbf{E} .
\end{aligned}
$$

From (3.5) it is easy to see that whenever $\mathbf{A}$ has distinct eigenvalues, $\alpha_{1}, \alpha_{2}$ must be isotropic in $\mathbf{A}$ and $\mathbf{E}$, and $\alpha_{3}$ must be isotropic in $\mathbf{A}$.

From $\mathbf{G}(\mathbf{A}, \lambda \mathbf{E})=\mathbf{H}(\mathbf{A}, \lambda \mathbf{E})-\alpha_{3}(\mathbf{A}) \lambda \mathbf{E}=\lambda\left(\mathbf{H}(\mathbf{A}, \mathbf{E})-\alpha_{3}(\mathbf{A}) \mathbf{E}\right)$, we see that

$$
\alpha_{1}(\mathbf{A}, \lambda \mathbf{E}) \mathbf{I}+\alpha_{2}(\mathbf{A}, \lambda \mathbf{E}) \mathbf{A}=\lambda \alpha_{1}(\mathbf{A}, \mathbf{E}) \mathbf{I}+\lambda \alpha_{2}(\mathbf{A}, \mathbf{E}) \mathbf{A} .
$$

Hence

$$
\alpha_{i}(\mathbf{A}, \lambda \mathbf{E})=\lambda \alpha_{i}(\mathbf{A}, \mathbf{E}) \quad(i=1,2)
$$

for all $\lambda \in \mathbb{R}^{1}, \mathbf{E} \in \mathrm{Sym}$, and $\mathbf{A}$ with distinct eigenvalues. Similarly we prove that

$$
\alpha_{i}\left(\mathbf{A}, \mathbf{E}_{1}\right)+\alpha_{i}\left(\mathbf{A}, \mathbf{E}_{2}\right)=\alpha_{i}\left(\mathbf{A}, \mathbf{E}_{1}+\mathbf{E}_{2}\right) \quad(i=1,2)
$$

for all $\mathbf{E}_{1}, \mathbf{E}_{2} \in \operatorname{Sym}$ and all $\mathbf{A}$ with distinct eigenvalues. 
Since $\alpha_{3}$ is an isotropic function of $\mathbf{A}$, it is a symmetric function of the eigenvalues $\lambda_{1}, \lambda_{2}$ of $\mathbf{A}$. Under a coordinate system where $\mathbf{A}$ and $\mathbf{E}$ assume the form (3.4), if we let

$$
\mathbf{H}=\left(\begin{array}{ll}
h_{11} & h_{12} \\
h_{12} & h_{22}
\end{array}\right)
$$

then

$$
\begin{array}{lr}
h_{11}=\alpha_{1}+\alpha_{2} \lambda_{1}+\alpha_{3} e_{11}, \\
h_{22}=\alpha_{1}+\alpha_{2} \lambda_{2}+\alpha_{3} e_{22}, \\
h_{12}=r \alpha_{3} e_{12} .
\end{array}
$$

Equation $(3.10)_{3}$ is valid even when $\lambda_{1}=\lambda_{2}$. Since $h_{12}$ is of class $C^{n+2}$ in $\lambda_{1}, \lambda_{2}, e_{11}, e_{12}$, and $e_{22}$ over $\mathbb{R}^{5}, \alpha_{3}$ is of class $C^{n+2}$ in $\lambda_{1}$ and $\lambda_{2}$ over $\mathbb{R}^{2}$. From (3.10) we obtain

$$
\begin{aligned}
& \alpha_{1}=\frac{1}{\lambda_{2}-\lambda_{1}}\left(h_{11} \lambda_{2}-h_{22} \lambda_{1}+\alpha_{3} e_{22} \lambda_{1}-\alpha_{3} e_{11} \lambda_{2}\right)=\frac{f_{1}\left(\lambda_{1}, \lambda_{2}, e_{11}, e_{12}, e_{22}\right)}{\lambda_{2}-\lambda_{1}}, \\
& \alpha_{2}=\frac{1}{\lambda_{2}-\lambda_{1}}\left(h_{22}-h_{11}+\alpha_{3} e_{11}-\alpha_{3} e_{22}\right)=\frac{f_{2}\left(\lambda_{1}, \lambda_{2}, e_{11}, e_{12}, e_{22}\right)}{\lambda_{2}-\lambda_{1}},
\end{aligned}
$$

where $f_{1}, f_{2} \in C^{n+2}$ over $\mathbb{R}^{5}$. Since $\alpha_{i}(i=1,2)$ is isotropic in $\mathbf{A}$ and $\mathbf{E}$, it assumes the same value whether we use the coordinate system where $\mathbf{A}$ is diagonal or that where $\mathbf{E}$ is diagonal. Moreover, since $\alpha_{1}$ and $\alpha_{2}$ are linear in $\mathbf{E}$, under the coordinate system where $\mathbf{E}=\operatorname{diag}\left[e_{1}, e_{2}\right]$, we have

$$
\begin{aligned}
& \alpha_{1}=C_{11}(\mathbf{A}) e_{1}+C_{12}(\mathbf{A}) e_{2}, \\
& \alpha_{2}=C_{21}(\mathbf{A}) e_{1}+C_{22}(\mathbf{A}) e_{2},
\end{aligned}
$$

for some coefficients $C_{i j}(i, j=1,2)$ defined for all $\mathbf{A}$ with distinct eigenvalues $\lambda_{1}, \lambda_{2}$. It follows from (3.11) that

$$
\begin{aligned}
& C_{11}(\mathbf{A}) e_{1}+C_{12}(\mathbf{A}) e_{2}=\frac{f_{1}\left(\lambda_{1}, \lambda_{2}, e_{11}, e_{12}, e_{22}\right)}{\lambda_{2}-\lambda_{1}}, \\
& C_{21}(\mathbf{A}) e_{1}+C_{22}(\mathbf{A}) e_{2}=\frac{f_{2}\left(\lambda_{1}, \lambda_{2}, e_{11}, e_{12}, e_{22}\right)}{\lambda_{2}-\lambda_{1}} .
\end{aligned}
$$

Now let $J_{0}=e_{1}+e_{2}=\operatorname{tr} \mathbf{E}$, and $J_{1}=a_{11} e_{1}+a_{22} e_{2}=\operatorname{tr}(\mathbf{A E})$; here $a_{i j}$ are the components of $\mathbf{A}$ under the coordinate system where $\mathbf{E}$ is diagonal. Note that

$$
\begin{aligned}
& a_{11}=\lambda_{1} \cos ^{2} \theta+\lambda_{2} \sin ^{2} \theta, \\
& a_{22}=\lambda_{1} \sin ^{2} \theta+\lambda_{2} \cos ^{2} \theta,
\end{aligned}
$$

where $\theta$ is the angle of rotation required to bring the coordinate system for which $\mathbf{E}$ is diagonal to that for which $\mathbf{A}$ is diagonal. We want $\alpha_{i j}$ such that

$$
\left(\begin{array}{l}
\alpha_{1} \\
\alpha_{2}
\end{array}\right)=\left(\begin{array}{ll}
\alpha_{11} & \alpha_{12} \\
\alpha_{21} & \alpha_{22}
\end{array}\right)\left(\begin{array}{l}
J_{0} \\
J_{1}
\end{array}\right)=\left(\begin{array}{ll}
\alpha_{11} & \alpha_{12} \\
\alpha_{21} & \alpha_{22}
\end{array}\right)\left(\begin{array}{cc}
1 & 1 \\
a_{11} & a_{22}
\end{array}\right)\left(\begin{array}{l}
e_{1} \\
e_{2}
\end{array}\right) \text {. }
$$


Comparing Eq. (3.15) with Eq. (3.12), we have for $\cos 2 \theta \neq 0$,

$$
\begin{aligned}
\left(\begin{array}{ll}
\alpha_{11} & \alpha_{12} \\
\alpha_{21} & \alpha_{22}
\end{array}\right) & =\left(\begin{array}{ll}
C_{11} & C_{12} \\
C_{21} & C_{22}
\end{array}\right)\left(\begin{array}{cc}
1 & 1 \\
a_{11} & a_{22}
\end{array}\right)^{-1} \\
& =\left(\begin{array}{ll}
C_{11} & C_{12} \\
C_{21} & C_{22}
\end{array}\right) \frac{1}{a_{22}-a_{11}}\left(\begin{array}{cc}
a_{22} & -1 \\
-a_{11} & 1
\end{array}\right) \\
& =\frac{1}{\left(\lambda_{2}-\lambda_{1}\right) \cos 2 \theta}\left(\begin{array}{lll}
C_{11} a_{22}-C_{12} a_{11} & -C_{11}+C_{12} \\
C_{21} a_{22}-C_{22} a_{11} & -C_{21}+C_{22}
\end{array}\right) .
\end{aligned}
$$

By putting $e_{1}=1, e_{2}=0$ in Eq. (3.13) and letting $e_{i j}$ assume their appropriate values (i.e., $e_{11}=\cos ^{2} \theta, e_{12}=\cos \theta \sin \theta, e_{22}=\sin ^{2} \theta$, where $\theta$ is the angle of rotation required to bring the coordinate system for which $\mathbf{E}$ is diagonal to that for which $\mathbf{A}$ is diagonal), we get

$$
C_{i 1}(\mathbf{A})=\frac{f_{i}\left(\lambda_{1}, \lambda_{2}, \cos ^{2} \theta, \cos \theta \sin \theta, \sin ^{2} \theta\right)}{\lambda_{2}-\lambda_{1}}
$$

for $i=1,2$. Similarly,

$$
C_{i 2}(\mathbf{A})=\frac{f_{i}\left(\lambda_{1}, \lambda_{2}, \sin ^{2} \theta,-\cos \theta \sin \theta, \cos ^{2} \theta\right)}{\lambda_{2}-\lambda_{1}}
$$

for $i=1,2$. It follows from Eq. (3.16) 3 that

$$
\begin{aligned}
& \alpha_{11}(\mathbf{A})= \\
& \frac{a_{22} f_{1}\left(\lambda_{1}, \lambda_{2}, \cos ^{2} \theta, \cos \theta \sin \theta, \sin ^{2} \theta\right)-a_{11} f_{1}\left(\lambda_{1}, \lambda_{2}, \sin ^{2} \theta,-\cos \theta \sin \theta, \cos ^{2} \theta\right)}{\left(\lambda_{2}-\lambda_{1}\right)^{2} \cos 2 \theta}
\end{aligned}
$$

where $a_{11}$ and $a_{22}$ are given by Eq. (3.14). Similarly, we obtain corresponding expressions for $\alpha_{12}, \alpha_{21}$, and $\alpha_{22}$.

Now, from the equation

$$
\mathbf{H}(\mathbf{A}, \mathbf{E})=\left(\alpha_{11} \operatorname{tr} \mathbf{E}+\alpha_{12} \operatorname{tr}(\mathbf{A E})\right) \mathbf{I}+\left(\alpha_{21} \operatorname{tr} \mathbf{E}+\alpha_{22} \operatorname{tr}(\mathbf{A E})\right) \mathbf{A}+\alpha_{3} \mathbf{E},
$$

we deduce from the isotropy of $\mathbf{H}$ that $\alpha_{i j}(i, j=1,2)$ are isotropic functions of A. Hence they are functions of $\lambda_{1}, \lambda_{2}$ alone and are independent of $\theta$. It follows from Eq. (3.19) that

$$
\alpha_{11}\left(\lambda_{1}, \lambda_{2}\right)=\frac{f_{11}\left(\lambda_{1}, \lambda_{2}\right)}{\left(\lambda_{2}-\lambda_{1}\right)^{2}}
$$

where $f_{11} \in C^{n+2}$ is symmetric in $\lambda_{1}$ and $\lambda_{2}$. Similarly,

$$
\alpha_{i j}\left(\lambda_{1}, \lambda_{2}\right)=\frac{f_{i j}\left(\lambda_{1}, \lambda_{2}\right)}{\left(\lambda_{2}-\lambda_{1}\right)^{2}} \quad(i, j=1,2)
$$

where $f_{i j} \in C^{n+2}$ is symmetric in $\lambda_{1}$ and $\lambda_{2}$.

Until now the functions $\alpha_{i j}$ were defined over the set $\left\{\left(\lambda_{1}, \lambda_{2}\right) \in \mathbb{R}^{2}: \lambda_{1} \neq \lambda_{2}\right\}$. The functions $f_{i j}$, however, are defined even when $\lambda_{1}=\lambda_{2}$. From Eqs. (3.14), 
(3.19), and (3.21), we obtain the formula

$$
f_{11}(\lambda, \lambda)=\lambda f_{1}(\lambda, \lambda, 1,0,0)-\lambda f_{1}(\lambda, \lambda, 0,0,1)
$$

by putting $\theta=0$. When $\mathbf{A}=\lambda \mathbf{I}, \mathbf{H}$ is a linear isotropic function of $\mathbf{E}$; so $\mathbf{H}(\lambda \mathbf{I}, \mathbf{E})=\beta(\lambda)(\operatorname{tr} \mathbf{E}) \mathbf{I}+\alpha_{3}(\lambda, \lambda) \mathbf{E}$ for some $\beta(\lambda) \in \mathbb{R}^{1}$. It follows then from the definition of $f_{1}$ (cf. Eq. (3.11)) that $f_{1}(\lambda, \lambda, 1,0,0)=f_{1}(\lambda, \lambda, 0,0,1)=0$. Hence, $f_{11}(\lambda, \lambda)=0$. Similarly we prove that $f_{i j}(\lambda, \lambda)=0$ for $i, j=1,2$. By Theorem 2.3 we conclude that $\alpha_{i j}$ may be extended by continuity to allow for $\lambda_{1}=\lambda_{2}$, and the resulting functions are of class $C^{n}$.

For a given $\mathbf{A}$, the tensor function $\mathbf{H}(\mathbf{A}, \cdot):$ Sym $\rightarrow$ Sym given by (3.2) is linear. Let us write $\mathbf{H}(\mathbf{A}, \cdot)=\mathbf{H}(\mathbf{A})[\cdot]$. For two second-order tensors $\mathbf{A}$ and $\mathbf{B}$, we define their dot product by $\mathbf{A} \cdot \mathbf{B}=\operatorname{tr}\left(\mathbf{A} \mathbf{B}^{\mathrm{T}}\right)$. The following corollary, which will be useful in some applications [9], follows from formula (3.2) after a straightforward calculation.

Corollary 3.2. Suppose furthermore that the isotropic tensor function $\mathbf{H}(\mathbf{A})[\cdot]$ in Theorem 3.1 satisfies

$$
\mathbf{E}_{1} \cdot \mathbf{H}(\mathbf{A})\left[\mathbf{E}_{2}\right]=\mathbf{H}(\mathbf{A})\left[\mathbf{E}_{1}\right] \cdot \mathbf{E}_{2}
$$

for all $\mathbf{E}_{1}$ and $\mathbf{E}_{2}$ in Sym. Then $\alpha_{12}=\alpha_{21}$.

Now, consider an isotropic function $\mathbf{G}:$ Sym $\rightarrow$ Sym. It is well known that $\mathbf{G}$ has a representation of the form

$$
\mathbf{G}(\mathbf{A})=\beta_{1} \mathbf{I}+\beta_{2} \mathbf{A},
$$

where $\beta_{1}$ and $\beta_{2}$ are symmetric functions of the repeated eigenvalues $\lambda_{1}, \lambda_{2}$ of $\mathbf{A}$. When $\lambda_{1} \neq \lambda_{2}, \beta_{1}$ and $\beta_{2}$ are uniquely determined by the formulae

$$
\beta_{1}=\frac{g_{1} \lambda_{2}-g_{2} \lambda_{1}}{\lambda_{2}-\lambda_{1}}, \quad \beta_{2}=\frac{g_{2}-g_{1}}{\lambda_{2}-\lambda_{1}},
$$

where $g_{1}$ and $g_{2}$ are the eigenvalues of $\mathbf{G} ; g_{1}$ and $g_{2}$ are functions of $\lambda_{1}, \lambda_{2}$, and $g_{1}\left(\lambda_{1}, \lambda_{2}\right)=g_{2}\left(\lambda_{2}, \lambda_{1}\right)$. When $\lambda_{1}=\lambda_{2}$, Eq. (3.25) no longer determines $\beta_{1}, \beta_{2}$ uniquely. Indeed the possible choices of the coefficients are infinite. It is easy to show by counterexample (cf. Truesdell and Noll [11, p. 33]; the exponent in their counterexample should read $-1 / 4$, not $1 / 4$ ) that even for a continuous $\mathbf{G}$, when $\lambda_{1}=\lambda_{2}$ there need not exist a choice of coefficients in (3.25) that will match with Eq. (3.26) to define continuous functions $\beta_{1}, \beta_{2}$ on $\mathbb{R}^{2}$. If $\mathbf{G}$ is of class $C^{1}$, however, it is known [5] that $\beta_{1}$ and $\beta_{2}$, as defined by Eq. (3.26) on $\mathbb{R}^{2} \backslash\left\{\left(\lambda_{1}, \lambda_{2}\right): \lambda_{1} \neq \lambda_{2}\right\}$ may be extended to be continuous functions on $\mathbb{R}^{2}$. Now, with Theorem 2.4 in hand, we can immediately strengthen the preceding extension theorem as follows:

THEOREM 3.3. Let Sym be the set of second-order symmetric tensors in two-dimensional space. Let $\mathbf{G}: \mathrm{Sym} \rightarrow \mathrm{Sym}, \mathbf{A} \mapsto \mathbf{G}(\mathbf{A})$, be isotropic and of class $C^{n+1}$ $(n \geq 0)$. Then there exist two $C^{n}$ functions $\beta_{1}, \beta_{2}: \mathbb{R}^{2} \rightarrow \mathbb{R}^{1}$, each of which is a symmetric function of the repeated eigenvalues of $\mathbf{A}$, such that

$$
\mathbf{G}(\mathbf{A})=\beta_{1} \mathbf{I}+\beta_{2} \mathbf{A} \text {. }
$$


Moreover, coefficient functions that satisfy Eq. (3.27) and enjoy the aforementioned continuity $(n=0)$ or differentiability $(n \geq 1)$ properties are unique.

Proof. The conclusion follows from Theorem 2.4 and the fact that $g_{1}$ and $g_{2}$ are of class $C^{n+1}$.

Acknowledgment. It was an REU Supplement to Kentucky NSF/EPSCoR Grant No. RII-8610671 that initially drew J. B. Schanding, then an undergraduate student, to take part in the research reported here. This research was subsequently supported in part with funds from the National Science Foundation (Grant No. EHR-9108764) and the Commonwealth of Kentucky through the Kentucky NSF/EPSCoR Advanced Development Program.

\section{REFERENCES}

[1] C.-C. Wang, A new representation theorem for isotropic functions. Part 1, Arch. Rational Mech. Anal. 36, 166-197 (1970)

[2] C.-C. Wang, A new representation theorem for isotropic functions. Part 2, Arch. Rational Mech. Anal. 36, 198-223 (1970)

[3] G. F. Smith, On isotropic functions of symmetric tensors, skew-symmetric tensors and vectors, Internat. J. Engrg. Sci. 9, 899-916 (1971)

[4] J. Serrin, The derivation of stress-deformation relations for a Stokesian fluid, J. Math. Mech. 8, 459-468 (1959)

[5] C.-S. Man, Remarks on the continuity of the scalar coefficients in the representation $\mathbf{H}(\mathbf{A})=\alpha \mathbf{I}+\beta \mathbf{A}$ $+\gamma \mathbf{A}^{2}$ for isotropic tensor functions, J. Elasticity 34, 229-238 (1994)

[6] J. M. Ball, Differentiability properties of symmetric and isotropic functions, Duke Math. J. 51, 699-728 (1984)

[7] R. S. Rivlin and J. L. Ericksen, Stress-deformation relations for isotropic materials, J. Rational Mech. Anal. 4, 323-424 (1955)

[8] W. Noll, On the continuity of the solid and fluid states, J. Rational Mech. Anal. 4, 3-81 (1955)

[9] C.-S. Man, On the acoustoelastic earing coefficient of plastically prestrained sineets, Proceedings of the 2nd International Conference on Nonlinear Mechanics, Wei-zang Chien et al. (eds.), Peking University Press, Beijing, China, 1993, pp. 66-71

[10] H. Whitney, Differentiability of the remainder term in Taylor's formula, Duke Math. J. 10, 153-158 (1943)

[11] C. Truesdell and W. Noll, The non-linear field theories of mechanics, vol. III/3 of S. Flügge's Encyclopedia of Physics, Springer-Verlag, Berlin, 1965

[12] C.-S. Man, Smoothness of the scalar coefficients in the representation $\mathbf{H}(\mathbf{A})=\alpha \mathbf{I}+\beta \mathbf{A}+\gamma \mathbf{A}^{2}$ for isotropic tensor functions of class $C^{r}$, J. Elasticity (to appear) 